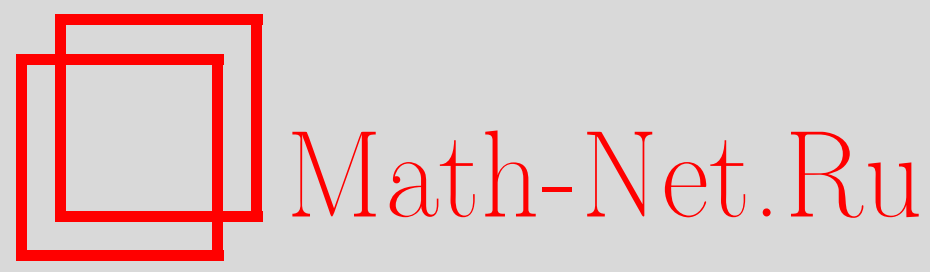

В. П. Маслов, О фазовом переходе для классических фермионов, Матем. заметки, 1998, том 64, выпуск 3, 470-473

DOI: https://doi.org/10.4213/mzm1420

Использование Общероссийского математического портала Math-Net.Ru подразумевает, что вы прочитали и согласны с пользовательским соглашением http://www.mathnet.ru/rus/agreement

Параметры загрузки:

IP : 3.89 .197 .203

26 апреля 2023 г., $11: 29: 32$

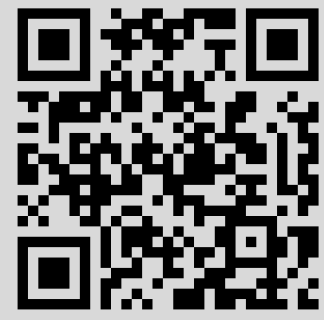




\section{О ФАЗОВОМ ПЕРЕХОДЕ ДЛЯ КЛАССИЧЕСКИХ ФЕРМИОНОВ}

\section{В. П. Маслов}

Рассмотрим уравнение Лиувилля для системы $N$ классических фермионов со спином, которое имеет вид

$$
\begin{aligned}
& \left(-i \sum_{j=1}^{N} p_{j} \frac{\partial}{\partial x_{j}}+i \sum_{j=1}^{N} \sum_{k=j+1}^{N}\left(\frac{\partial V\left(x_{j}-x_{k} ; p_{j}, p_{k} ; s_{j}, s_{k}\right)}{\partial x_{j}} \frac{\partial}{\partial p_{j}}\right.\right. \\
& +\frac{\partial V\left(x_{j}-x_{k} ; p_{j}, p_{k} ; s_{j}, s_{k}\right)}{\partial x_{k}} \frac{\partial}{\partial p_{k}}-\frac{\partial V\left(x_{j}-x_{k} ; p_{j}, p_{k} ; s_{j}, s_{k}\right)}{\partial p_{j}} \frac{\partial}{\partial x_{j}} \\
& \left.\left.-\frac{\partial V\left(x_{j}-x_{k} ; p_{j}, p_{k} ; s_{j}, s_{k}\right)}{\partial p_{k}} \frac{\partial}{\partial x_{k}}\right)\right) \rho\left(x_{1}, p_{1}, s_{1} ; \ldots ; x_{N}, p_{N}, s_{N}\right) \\
& \quad=\lambda \rho\left(x_{1}, p_{1}, s_{1} ; \ldots ; x_{N}, p_{N}, s_{N}\right),
\end{aligned}
$$

где $x_{j}, p_{j} \in \mathbb{R}^{3} ; s_{j}=1,2$ - спиновые переменные; $V\left(x ; p_{1}, p_{2} ; s_{1}, s_{2}\right)=V\left(-x ; p_{2}, p_{1} ; s_{2}, s_{1}\right) \in C^{1}$; $\rho\left(x_{1}, p_{1}, s_{1} ; \ldots ; x_{N}, p_{N}, s_{N}\right)$ - антисимметричная функция относительно перестановки аргументов $\left(x_{j}, p_{j}, s_{j}\right),\left(x_{k}, p_{k}, s_{k}\right)$ и использованы обозначения типа

$$
p \frac{\partial}{\partial x}=\sum_{\alpha=1}^{3} p^{\alpha} \frac{\partial}{\partial x^{\alpha}} .
$$

Антисимметричные решения уравнения (1) при четных $N$ выражаются через собственные функции вторично квантованного оператора вида

$$
\begin{aligned}
& \widehat{H}_{N}=\sum_{s_{1}=1}^{2} \cdots \sum_{s_{N}=1}^{2} \int \cdots \int d x_{1} d p_{1} \ldots d x_{N} d p_{N} \widehat{B}^{+}\left(x_{1}, p_{1}, s_{1} ; x_{2}, p_{2}, s_{2}\right) \cdots \\
& \quad \times \widehat{B}^{+}\left(x_{N-1}, p_{N-1}, s_{N-1} ; x_{N}, p_{N}, s_{N}\right)\left(-i \sum_{j=1}^{N} p_{j} \frac{\partial}{\partial x_{j}}\right. \\
& \quad+i \sum_{j=1}^{N} \sum_{k=j+1}^{N}\left(\frac{\partial V\left(x_{j}-x_{k} ; p_{j}, p_{k} ; s_{j}, s_{k}\right)}{\partial x_{j}} \frac{\partial}{\partial p_{j}}+\frac{\partial V\left(x_{j}-x_{k} ; p_{j}, p_{k} ; s_{j}, s_{k}\right)}{\partial x_{k}} \frac{\partial}{\partial p_{k}}\right. \\
& \left.\left.\quad-\frac{\partial V\left(x_{j}-x_{k} ; p_{j}, p_{k} ; s_{j}, s_{k}\right)}{\partial p_{j}} \frac{\partial}{\partial x_{j}}-\frac{\partial V\left(x_{j}-x_{k} ; p_{j}, p_{k} ; s_{j}, s_{k}\right)}{\partial p_{k}} \frac{\partial}{\partial x_{k}}\right)\right) \\
& \quad \times \underset{x_{1}, p_{1}, s_{1} ; \ldots ; x_{N}, p_{N}, s_{N}}{\operatorname{Asymm}} \widehat{B}^{-}\left(x_{1}, p_{1}, s_{1} ; x_{2}, p_{2}, s_{2}\right) \cdots \widehat{B}^{-}\left(x_{N-1}, p_{N-1}, s_{N-1} ; x_{N}, p_{N}, s_{N}\right)
\end{aligned}
$$

где

$$
\begin{gathered}
\underset{x_{1}, p_{1}, s_{1} ; \ldots ; x_{N}, p_{N}, s_{N}}{\operatorname{Asymm}} f\left(x_{1}, p_{1}, s_{1} ; \ldots ; x_{N}, p_{N}, s_{N}\right) \\
=\sum_{\sigma}(-1)^{\operatorname{sign} \sigma}\left(\widehat{P}_{\sigma} f\right)\left(x_{1}, p_{1}, s_{1} ; \ldots ; x_{N}, p_{N}, s_{N}\right)
\end{gathered}
$$

Работа выполнена при частичной поддержке Российского фонда фундаментальных исследований, грант № 96-01-01544. 
и сумма берется по всем перестановкам $\sigma$ аргументов $\left(x_{j}, p_{j}, s_{j}\right), \operatorname{sign} \sigma-$ знак перестановки, $\widehat{P}_{\sigma}-$ оператор перестановки аргументов, операторы $\widehat{B}^{ \pm}\left(x_{1}, p_{1}, s_{1} ; x_{2}, p_{2}, s_{2}\right)$ удовлетворяют коммутационным соотношениям вида

$$
\begin{aligned}
& {\left[\widehat{B}^{-}\left(x_{1}, p_{1}, s_{1} ; x_{2}, p_{2}, s_{2}\right), \widehat{B}^{+}\left(x_{1}^{\prime}, p_{1}^{\prime}, s_{1}^{\prime} ; x_{2}^{\prime}, p_{2}^{\prime}, s_{2}^{\prime}\right)\right]} \\
& \quad=\delta\left(x_{1}-x_{1}^{\prime}\right) \delta\left(p_{1}-p_{1}^{\prime}\right) \delta\left(x_{2}-x_{2}^{\prime}\right) \delta\left(p_{2}-p_{2}^{\prime}\right) \delta_{s_{1}} s_{1}^{\prime} \delta_{s_{2}} s_{2}^{\prime} \\
& \quad\left[\widehat{B}^{ \pm}\left(x_{1}, p_{1}, s_{1} ; x_{2}, p_{2}, s_{2}\right), \widehat{B}^{ \pm}\left(x_{1}^{\prime}, p_{1}^{\prime}, s_{1}^{\prime} ; x_{2}^{\prime}, p_{2}^{\prime}, s_{2}^{\prime}\right)\right]=0
\end{aligned}
$$

В квазиклассическом пределе, когда коммутаторы между операторами $\widehat{B}^{+}$и $\widehat{B}^{-}$малы, оператору (2) отвечает бесконечномерная гамильтонова система уравнений вида

$$
\begin{aligned}
& \sum_{s_{3}=1} \cdots \sum_{s_{N}=1} \int \cdots \int d x_{3} d p_{3} \cdots d x_{N} d p_{N} \Phi^{*}\left(x_{3}, p_{3}, s_{3} ; x_{4}, p_{4}, s_{4}\right) \cdots \\
& \times \Phi^{*}\left(x_{N-1}, p_{N-1}, s_{N-1} ; x_{N}, p_{N}, s_{N}\right)\left(-i \sum_{j=1}^{N} p_{j} \frac{\partial}{\partial x_{j}}\right. \\
& \quad+i \sum_{j=1}^{N} \sum_{k=j+1}^{N}\left(\frac{\partial V\left(x_{j}-x_{k} ; p_{j}, p_{k} ; s_{j}, s_{k}\right)}{\partial x_{j}} \frac{\partial}{\partial p_{j}}+\frac{\partial V\left(x_{j}-x_{k} ; p_{j}, p_{k} ; s_{j}, s_{k}\right)}{\partial x_{k}} \frac{\partial}{\partial p_{k}}\right. \\
& \left.\left.\quad-\frac{\partial V\left(x_{j}-x_{k} ; p_{j}, p_{k} ; s_{j}, s_{k}\right)}{\partial p_{j}} \frac{\partial}{\partial x_{j}}-\frac{\partial V\left(x_{j}-x_{k} ; p_{j}, p_{k} ; s_{j}, s_{k}\right)}{\partial p_{k}} \frac{\partial}{\partial x_{k}}\right)-\lambda\right) \\
& \times \underset{\substack{\mathrm{Asymm} \\
x_{1}, p_{1}, s_{1} ; \ldots ; x_{N}, p_{N}, s_{N}}}{\Phi}\left(x_{1}, p_{1}, s_{1} ; x_{2}, p_{2}, s_{2}\right) \cdots \Phi\left(x_{N-1}, p_{N-1}, s_{N-1} ; x_{N}, p_{N}, s_{N}\right),
\end{aligned}
$$

где $\Phi\left(x_{1}, p_{1}, s_{1} ; x_{2}, p_{2}, s_{2}\right)=-\Phi\left(x_{2}, p_{2}, s_{2} ; x_{1}, p_{1}, s_{1}\right)$. Аналогично работе [1], суммируя эти уравнения по числу фермионов $N$, мы получим уравнения, описывающие систему классических фермионов:

$$
\begin{aligned}
& -i\left(p_{1} \frac{\partial}{\partial x_{1}}+p_{2} \frac{\partial}{\partial x_{2}}\right) G\left(x_{1}, p_{1}, s_{1} ; x_{2}, p_{2}, s_{2}\right) \\
& +i \sum_{s^{\prime}=1}^{2} \iint d x^{\prime} d p^{\prime}\left(\frac{\partial V\left(x_{1}-x^{\prime} ; p_{1}^{3}, p^{\prime} ; s_{1}, s^{\prime}\right)}{\partial x_{1}} \frac{\stackrel{2}{\partial}}{\partial p_{1}}+\frac{\partial V\left(x_{1}-x^{\prime} ; p_{1}, p^{\prime} ; s_{1}, s^{\prime}\right)}{\partial x^{\prime}} \frac{\stackrel{2}{\partial}}{\partial p^{\prime}}\right. \\
& \left.-\frac{\partial V\left(\stackrel{3}{x_{1}}-x^{\prime} ; p_{1}, p^{\prime} ; s_{1}, s^{\prime}\right)}{\partial p_{1}} \frac{\stackrel{2}{\partial}}{\partial x_{1}}-\frac{\partial V\left(x_{1}-\stackrel{3}{x}^{\prime} ; p_{1}, p^{\prime} ; s_{1}, s^{\prime}\right)}{\partial p^{\prime}} \frac{\stackrel{2}{\partial}}{\partial x^{\prime}}\right)
\end{aligned}
$$

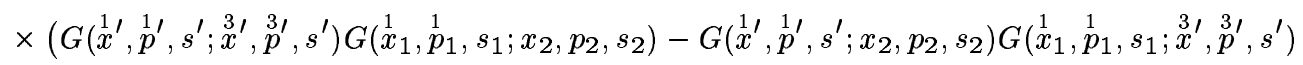

$$
\begin{aligned}
& \left.+R^{*}\left(\stackrel{1}{x}^{\prime}, \stackrel{1}{p}^{\prime}, s^{\prime} ; \stackrel{1}{x}_{1}, \stackrel{1}{p}_{1}, s_{1}\right) R\left(\stackrel{3}{x^{\prime}}, \stackrel{3}{p}^{\prime}, s^{\prime} ; x_{2}, p_{2}, s_{2}\right)\right) \\
& +i \sum_{s^{\prime}=1}^{2} \iint d x^{\prime} d p^{\prime}\left(\frac{\partial V\left(x_{2}-x^{\prime} ; p_{2}, p^{\prime} ; s_{2}, s^{\prime}\right)}{\partial x_{2}} \frac{\stackrel{2}{\partial}}{\partial p_{2}}+\frac{\partial V\left(x_{2}-x^{\prime} ; p_{2}, p^{\prime} ; s_{2}, s^{\prime}\right)}{\partial x^{\prime}} \frac{\stackrel{2}{\partial}}{\partial p^{\prime}}\right. \\
& \left.-\frac{\partial V\left(\stackrel{3}{x_{2}}-x^{\prime} ; p_{2}, p^{\prime} ; s_{2}, s^{\prime}\right)}{\partial p_{2}} \frac{\stackrel{2}{\partial}}{\partial x_{2}}-\frac{\partial V\left(x_{2}-\stackrel{3}{x}^{\prime} ; p_{2}, p^{\prime} ; s_{2}, s^{\prime}\right)}{\partial p^{\prime}} \frac{\stackrel{2}{\partial}}{\partial x^{\prime}}\right) \\
& \times\left(G\left(\stackrel{3}{x}^{\prime}, \stackrel{3}{p}^{\prime}, s^{\prime} ; \stackrel{1}{x}^{\prime}, \stackrel{1}{p}^{\prime}, s^{\prime}\right) G\left(x_{1}, p_{1}, s_{1} ; \stackrel{1}{x}_{2}, \stackrel{1}{p}_{2}, s_{2}\right)-G\left(\stackrel{3}{x}^{\prime}, \stackrel{3}{p}^{\prime}, s^{\prime} ; \stackrel{1}{x}_{2}, \stackrel{1}{p_{2}}, s_{2}\right) G\left(x_{1}, p_{1}, s_{1} ; \stackrel{1}{x}^{\prime}, \stackrel{1}{p}^{\prime}, s^{\prime}\right)\right. \\
& \left.+R^{*}\left(\stackrel{3}{x}, \stackrel{3}{p}^{\prime}, s^{\prime} ; x_{1}, p_{1}, s_{1}\right) R\left(\stackrel{1}{x}, \stackrel{1}{p}^{\prime}, s^{\prime} ; \stackrel{1}{x_{2}}, \stackrel{1}{p_{2}}, s_{2}\right)\right)=0
\end{aligned}
$$




$$
\begin{aligned}
& -i\left(p_{1} \frac{\partial}{\partial x_{1}}+p_{2} \frac{\partial}{\partial x_{2}}\right) R\left(x_{1}, p_{1}, s_{1} ; x_{2}, p_{2}, s_{2}\right) \\
& +i\left(\frac{\partial V\left(x_{1}-x_{2} ; p_{1}, p_{2} ; s_{1}, s_{2}\right)}{\partial x_{1}} \frac{\partial}{\partial p_{1}}+\frac{\partial V\left(x_{1}-x_{2} ; p_{1}, p_{2} ; s_{1}, s_{2}\right)}{\partial x_{2}} \frac{\partial}{\partial p_{2}}\right. \\
& \left.-\frac{\partial V\left(x_{1}-x_{2} ; p_{1}, p_{2} ; s_{1}, s_{2}\right)}{\partial p_{1}} \frac{\partial}{\partial x_{1}}-\frac{\partial V\left(x_{1}-x_{2} ; p_{1}, p_{2} ; s_{1}, s_{2}\right)}{\partial p_{2}} \frac{\partial}{\partial x_{2}}\right) \\
& \times R\left(x_{1}, p_{1}, s_{1} ; x_{2}, p_{2}, s_{2}\right) \\
& +i \sum_{s^{\prime}=1}^{2} \iint d x^{\prime} d p^{\prime}\left(\frac{\partial V\left(x_{1}-x^{\prime} ; \stackrel{3}{p}_{1}, p^{\prime} ; s_{1}, s^{\prime}\right)}{\partial x_{1}} \frac{\stackrel{2}{\partial}}{\partial p_{1}}+\frac{\partial V\left(x_{1}-x^{\prime} ; p_{1}, \stackrel{3}{p}^{\prime} ; s_{1}, s^{\prime}\right)}{\partial x^{\prime}} \frac{\stackrel{2}{\partial}}{\partial p^{\prime}}\right. \\
& -\frac{\partial V\left(\stackrel{3}{x}_{1}-x^{\prime} ; p_{1}, p^{\prime} ; s_{1}, s^{\prime}\right)}{\partial p_{1}} \frac{\stackrel{2}{\partial}}{\partial x_{1}}-\frac{\partial V\left(x_{1}-\stackrel{3}{x}^{\prime} ; p_{1}, p^{\prime} ; s_{1}, s^{\prime}\right)}{\partial p^{\prime}} \frac{\stackrel{2}{\partial}}{\partial x^{\prime}} \\
& +\frac{\partial V\left(x_{2}-x^{\prime} ; \stackrel{3}{p}_{2}, p^{\prime} ; s_{2}, s^{\prime}\right)}{\partial x_{2}} \frac{\stackrel{2}{\partial}}{\partial p_{2}}+\frac{\partial V\left(x_{2}-x^{\prime} ; p_{2}, \stackrel{3}{p}^{\prime} ; s_{2}, s^{\prime}\right)}{\partial x^{\prime}} \frac{\stackrel{2}{\partial}}{\partial p^{\prime}} \\
& \left.-\frac{\partial V\left(\stackrel{3}{x}_{2}-x^{\prime} ; p_{2}, p^{\prime} ; s_{2}, s^{\prime}\right)}{\partial p_{2}} \frac{\stackrel{2}{\partial}}{\partial x_{2}}-\frac{\partial V\left(x_{2}-\stackrel{3}{x}^{\prime} ; p_{2}, p^{\prime} ; s_{2}, s^{\prime}\right)}{\partial p^{\prime}} \frac{\stackrel{2}{\partial}}{\partial x^{\prime}}\right) \\
& \times\left(G\left(\stackrel{3}{x}^{\prime}, \stackrel{3}{p}^{\prime}, s^{\prime} ; \stackrel{1}{x}^{\prime}, \stackrel{1}{p}^{\prime}, s^{\prime}\right) R\left(\stackrel{1}{x}_{1}, \stackrel{1}{p}_{1}, s_{1} ; \stackrel{1}{x}_{2}, \stackrel{1}{p}_{2}, s_{2}\right)-G\left(\stackrel{3}{x}^{\prime}, \stackrel{3}{p}^{\prime}, s^{\prime} ; \stackrel{1}{x}_{1}, \stackrel{1}{p}_{1}, s_{1}\right) R\left(\stackrel{1}{x}^{\prime}, \stackrel{1}{p}^{\prime}, s^{\prime} ; \stackrel{1}{x}_{2}, \stackrel{1}{p}_{2}, s_{2}\right)\right. \\
& \left.+G\left(\stackrel{3}{x}^{\prime}, \stackrel{3}{p}^{\prime}, s^{\prime} ; \stackrel{1}{x}_{2}, \stackrel{1}{p}_{2}, s_{2}\right) R\left(\stackrel{1}{x}^{\prime}, \stackrel{1}{p}^{\prime}, s^{\prime} ; \stackrel{1}{x}_{1}, \stackrel{1}{p}_{1}, s_{1}\right)\right)=0
\end{aligned}
$$

где индексы над операторами обозначают порядок действия [2], $G\left(x_{1}, p_{1}, s_{1} ; x_{2}, p_{2}, s_{2}\right)$ и $R\left(x_{1}, p_{1}\right.$, $\left.s_{1} ; x_{2}, p_{2}, s_{2}\right)$ удовлетворяют соотношениям

$$
\begin{aligned}
& G\left(x_{1}, p_{1}, s_{1} ; x_{2}, p_{2}, s_{2}\right)=G^{*}\left(x_{2}, p_{2}, s_{2} ; x_{1}, p_{1}, s_{1}\right) \\
& R\left(x_{1}, p_{1}, s_{1} ; x_{2}, p_{2}, s_{2}\right)=-R\left(x_{2}, p_{2}, s_{2} ; x_{1}, p_{1}, s_{1}\right) .
\end{aligned}
$$

Рассмотрим решения уравнений (4), (5), которые отвечают температурным состояниям системы классических фермионов. Температурное решение уравнения (1) есть интеграл по энергии $E$ распределения Гиббса и функций $\widetilde{\rho}_{E}\left(x_{1}, p_{1}, s_{1} ; \ldots ; x_{N}, p_{N}, s_{N}\right)$, которые являются пределом при $h \rightarrow 0$ ( $h$ - постоянная Планка) функций

$$
\begin{aligned}
\widetilde{\rho}_{E}^{h}\left(x_{1}, p_{1}, s_{1} ; \ldots ; x_{N}, p_{N}, s_{N}\right)= & \Psi_{E}\left(x_{1}, s_{1} ; \ldots ; x_{N}, s_{N}\right) \widetilde{\Phi}_{E}^{*}\left(p_{1}, \ldots, p_{N}\right) \\
& \times \exp \left(-\frac{i}{h} \sum_{j=1} N p_{j} x_{j}\right) \frac{1}{(2 \pi h)^{3 N / 2}},
\end{aligned}
$$

где антисимметричные функции $\Psi_{E}\left(x_{1}, s_{1} ; \ldots ; x_{N}, s_{N}\right)$ - решения отвечающего уравнению (1) уравнения Шрёдингера для фермионов со спином, симметричные функции $\widetilde{\Phi}_{E}\left(p_{1}, \ldots, p_{N}\right)-$ решения уравнения Шрёдингера для бозонов в импульсном представлении, которые сосредоточены на уровне энергии $E$. При этом на каждом уровне энергии фермионные функции распределены по Гиббсу, а бозонная функция на каждом уровне только одна. Такому случаю отвечают решения уравнений (4), (5), имеюшие вид

$$
\begin{gathered}
R\left(x_{1}, p_{1}, s_{1} ; x_{2}, p_{2}, s_{2}\right)=0 \\
G\left(x_{1}, p_{1}, s_{1} ; x_{2}, p_{2}, s_{2}\right)=\delta\left(x_{1}-x_{2}\right) \delta\left(p_{1}-p_{2}\right) \delta_{s_{1}} s_{2} \frac{1}{e^{\left(\omega\left(p_{1}\right)-\mu\right) / \theta}+1},
\end{gathered}
$$

где $\theta$ - температура, $\mu$ - химический потенциал, $\omega(p)$ является решением уравнения

$$
\omega(p)=\frac{p^{2}}{2}+2 \iint d y d k V(x-y ; p, k) \frac{1}{e^{(\omega(k)-\mu) / \theta}+1} .
$$


Решения вида (6) отвечают температурному состоянию "холостых" классических фермионов. Согласно общей концепции [1], переход в "парное" состояние происходит при температуре, при которой от решений вида (6) ответвляются решения уравнений $(4),(5)$ с $R\left(x_{1}, p_{1}, s_{1} ; x_{2}, p_{2}, s_{2}\right) \neq 0$. Наложим на потенциал взаимодействия $V\left(x ; p_{1}, p_{2}\right)$ условие

$$
\iint d y d k \frac{\partial V(x-y ; p, k)}{\partial p} \frac{1}{e^{(\omega(k)-\mu) / \theta}+1}=0,
$$

справедливое при любых $\theta, \mu$. В этом случае для системы классических фермионов температура фазового перехода в сверхпроводящее состояние определяется из уравнения в вариациях для уравнения (5), которое имеет вид

$$
\begin{aligned}
& -i\left(p_{1} \frac{\partial}{\partial x_{1}}+p_{2} \frac{\partial}{\partial x_{2}}\right) \Phi\left(x_{1}, p_{1}, s_{1} ; x_{2}, p_{2}, s_{2}\right) \\
& +i\left(\frac{\partial V\left(x_{1}-x_{2} ; p_{1}, p_{2} ; s_{1}, s_{2}\right)}{\partial x_{1}} \frac{\partial}{\partial p_{1}}+\frac{\partial V\left(x_{1}-x_{2} ; p_{1}, p_{2} ; s_{1}, s_{2}\right)}{\partial x_{2}} \frac{\partial}{\partial p_{2}}\right. \\
& \left.-\frac{\partial V\left(x_{1}-x_{2} ; p_{1}, p_{2} ; s_{1}, s_{2}\right)}{\partial p_{1}} \frac{\partial}{\partial x_{1}}-\frac{\partial V\left(x_{1}-x_{2} ; p_{1}, p_{2} ; s_{1}, s_{2}\right)}{\partial p_{2}} \frac{\partial}{\partial x_{2}}\right) \\
& \times \Phi\left(x_{1}, p_{1}, s_{1} ; x_{2}, p_{2}, s_{2}\right) \\
& +i \sum_{s^{\prime}=1}^{2} \iint d x^{\prime} d p^{\prime}\left(\frac{\partial V\left(x_{1}-x^{\prime} ; p_{1}, p^{\prime} ; s_{1}, s^{\prime}\right)}{\partial x_{1}} \frac{\stackrel{2}{\partial}}{\partial p_{1}}+\frac{\partial V\left(x_{1}-x^{\prime} ; p_{1}, \stackrel{3}{p}^{\prime} ; s_{1}, s^{\prime}\right)}{\partial x^{\prime}} \frac{\stackrel{2}{\partial}}{\partial p^{\prime}}\right. \\
& -\frac{\partial V\left(\stackrel{3}{x}_{1}-x^{\prime} ; p_{1}, p^{\prime} ; s_{1}, s^{\prime}\right)}{\partial p_{1}} \frac{\stackrel{2}{\partial}}{\partial x_{1}}-\frac{\partial V\left(x_{1}-\stackrel{3}{x}^{\prime} ; p_{1}, p^{\prime} ; s_{1}, s^{\prime}\right)}{\partial p^{\prime}} \frac{\stackrel{2}{\partial}}{\partial x^{\prime}} \\
& +\frac{\partial V\left(x_{2}-x^{\prime} ; \stackrel{3}{p}_{2}, p^{\prime} ; s_{2}, s^{\prime}\right)}{\partial x_{2}} \frac{\stackrel{2}{\partial}}{\partial p_{2}}+\frac{\partial V\left(x_{2}-x^{\prime} ; p_{2}, \stackrel{3}{p}^{\prime} ; s_{2}, s^{\prime}\right)}{\partial x^{\prime}} \frac{\stackrel{2}{\partial}}{\partial p^{\prime}} \\
& \left.-\frac{\partial V\left(\stackrel{3}{x}_{2}-x^{\prime} ; p_{2}, p^{\prime} ; s_{2}, s^{\prime}\right)}{\partial p_{2}} \frac{\stackrel{2}{\partial}}{\partial x_{2}}-\frac{\partial V\left(x_{2}-\stackrel{3}{x}^{\prime} ; p_{2}, p^{\prime} ; s_{2}, s^{\prime}\right)}{\partial p^{\prime}} \frac{\stackrel{2}{\partial}}{\partial x^{\prime}}\right) \\
& \times\left(G\left(\stackrel{3}{x}^{\prime}, \stackrel{3}{p}^{\prime}, s^{\prime} ; \stackrel{1}{x}_{2}, \stackrel{1}{p}_{2}, s_{2}\right) \Phi\left(\stackrel{1}{x}, \stackrel{1}{p}^{\prime}, s^{\prime} ; \stackrel{1}{x}_{1}, \stackrel{1}{p}_{1}, s_{1}\right)\right.
\end{aligned}
$$

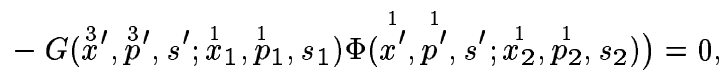

где $G\left(x_{1}, p_{1}, s_{1} ; x_{2}, p_{2}, s_{2}\right)$ имеет вид (6). Фазовый переход в системе с модельным потенциалом взаимодействия, который удовлетворяет условию (7), происходит при температуре, при которой у уравнения (8) появляется "нетривиальне" в некотором смысле решение

$$
\Phi\left(x_{1}, p_{1}, s_{1} ; x_{2}, p_{2}, s_{2}\right)=-\Phi\left(x_{2}, p_{2}, s_{2} ; x_{1}, p_{1}, s_{1}\right) .
$$

Пусть

$$
V\left(x_{1}-x_{2} ; p_{1}, p_{2} ; s_{1}, s_{2}\right)= \begin{cases}W\left(x_{1}-x_{2} ; p_{1}, p_{2}\right) \in C^{1} & \text { при } s_{1} \neq s_{2}, \\ 0 & \text { при } s_{1}=s_{2} .\end{cases}
$$

В этом случае уравнение (8) примет вид

$$
\left\{\frac{p_{1}^{2}}{2}+\frac{p_{2}^{2}}{2} ; \Phi\left(x_{1}, p_{1} ; x_{2}, p_{2}\right)\right\}+\left(1-n\left(p_{1}\right)-n\left(p_{2}\right)\right)\left\{W\left(x_{1}-x_{2} ; p_{1}, p_{2}\right) ; \Phi\left(x_{1}, p_{1} ; x_{2}, p_{2}\right)\right\}=0
$$

где $\{\cdot ; \cdot\}$ - скобка Пуассона, $n(p)=1 /\left(e^{(\omega(p)-\mu) / \theta}+1\right)$.

\section{СПИСОК ЦИТИРОВАННОЙ ЛИТЕРАТУРЫ}

1. Маслов В. П. // Матем. заметки.. Т. 64. № 2. С. 315-317. 2. Маслов В. П. Операторные методы. М.: Наука, 1973. 